\title{
Erratum: Carbon Honeycomb High Capacity Storage for Gaseous and Liquid Species [Phys. Rev. Lett. 116, 055501 (2016)]
}

Nina V. Krainyukova and Evgeniy N. Zubarev

(Received 16 November 2016; published 9 January 2017)

DOI: 10.1103/PhysRevLett.118.029901

The carbon honeycomb structure with high gas absorption capacity proposed in our Letter is built on a basis of averaged interatomic distances and angles derived from our TEM and diffraction measurements. But local arrangements can be corrected allowing for difference in atomic surrounding in walls and along junction lines. According to [1] the structures relaxed along the junction lines could be such as shown in Fig. 1. But as it was noticed in this theoretical paper vertical bonds along the junction are not absolutely stable and can be broken and recombine repeatedly. Such relaxation results in $s p^{3}$ bonding along these lines while the walls are still pure graphene ribbons with exclusively $s p^{2}$ bonding. This reference was omitted in our Letter. The relevant structural changes concern only two atoms involved in the alternation along a junction line out of 14 atoms in the initial elementary cell (for $n=1$ ). These two atoms are repeatedly slightly shifted either towards their neighbors or in the opposite direction. Such small dynamical structural transformations may hardly be detected by the structural methods only (especially if we study random structures) and other experimental techniques like Raman spectroscopy or high resolution atomic scale electron microscopy are needed. Such corrections do not change the main experimental findings reported in the Letter, i.e., the overall honeycomb configuration, wall edges and orientations, absorption levels, densities, etc. remain unaffected.

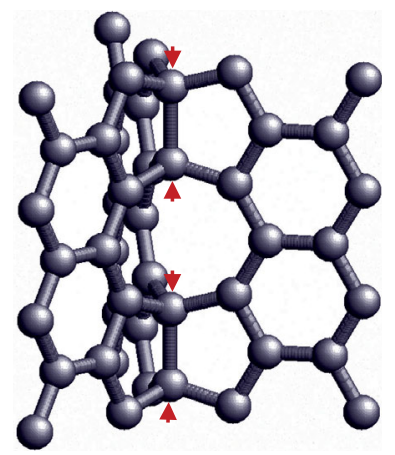

FIG. 1. The structure relaxed along the junction line [1].

We should also mention one technical misprint. The equation in the caption of Fig. 3 should read $S=4 \pi \sin \theta / \lambda$ without parentheses. This misprint does not affect the conclusions of the Letter either.

[1] T. Kawai, S. Okada, Y. Miyamoto, and A. Oshiyama, Phys. Rev. B 72, 035428 (2005). 